\title{
Theoretical Investigations on the Resonance Characteristics of CPW-Fed Miniaturized Strip Monopole Antennas
}

\author{
Amartya BANERJEE, Kaushik PATRA, Sayan CHATTERJEE, \\ Bhaskar GUPTA, Anup Kumar BANDYOPADHYAY \\ Dept. of ETCE, Jadavpur University, 188 Raja S. C. Mallick Road, Kolkata - 700032, India \\ amartyapotter@gmail.com, kaushikpatra294@gmail.com, sayan1234@gmail.com, \\ gupta_bh@yahoo.com, akbandyopadhyay@gmail.com
}

Submitted April 27, 2018 / Accepted August 16, 2018

\begin{abstract}
Novel closed form expressions to investigate $C P W$-fed miniaturized planar monopole antennas are presented. The analysis of a CPW-fed strip monopole structure is further extended to derive expressions for the input impedances of miniaturized strip-monopoles supporting slow wave propagation. The concept includes the modification of the propagation constants of the structures and the modeling of them into their equivalent straight rectangular strip-monopoles on planar substrates. The meandered/miniaturized strip-monopole radiators are disintegrated to model them as the superposition of simpler structures and circuit theoretic analyses are performed. The correctness of the reported expressions is validated against the simulations as well as the experimental measurements. Such closed-form analysis facilitates simpler design procedures against other available techniques such as conformal mapping and full wave analysis. The circuit theoretic approach reduces the computational requirements as against the available commercial EM solvers and provides a suitable interface platform for system analysis.
\end{abstract}

\section{Keywords}

CPW-fed miniaturized monopole antennas, resonance characteristics prediction, closed form expressions, induced EMF method, circuit model analysis

\section{Introduction}

With the advent of the ubiquitous wireless communication technologies along with the cutting-edge precision of fabrication, antenna miniaturization has become the focal point of electromagnetic researches in the recent years. Particularly, in medical applications, home and office appliances like the internet dongles and mobile handsets - the need for highly accurate and compact antennas, with greater reliability and lesser space requirements have become the primary topics of interests today. Implantable applications for medical requirements, [1], [2] facilitate the use of meandered antennas for miniaturization of the components. A meandered monopole antenna with shorted parasitic strips to yield a triple-band response was reported in [3] for WLAN and UMTS applications. Compact stripmonopole antennas for dongle and WLAN applications [4-6], Yagi-Uda radiator with CPW-fed strip monopole structures [7] and a CPW-fed strip monopole antenna for multiple system integration [8] are reported in recent works. These literatures highlight the importance of investigating strip-monopole structures and their meandered/miniaturized counterparts to develop theoretical knowledge for accurate designs.

Despite being so widely used for decades - the complete impedance characteristics even for the thin wire dipoles remain elusive till date [9] and further scopes of investigation are abundant. In [9], it is also reported that the different simulator and solver packages available for electromagnetic researches lead to different impedance values for dipole structures and the accuracy of the results depends on the numerical techniques used by the packages. These techniques [10-13] are complex and they offer geometry-dependent-convergence as described in [9] along with additional requirement of computational resources.

In this article a simple yet novel technique is proposed to analyze the $\mathrm{CPW}$-fed structures by considering the feed as a $50 \Omega$ transmission line and by modeling the miniaturized structures into their equivalent strip monopole configurations. The two miniaturized structures reported in this work are a meandered zigzag-shaped antenna configuration which is a planar counterpart of a normal mode helix and a planar corrugated monopole radiator inspired by the multiple meander-strip monopole structure as in [14]. It is also shown that they have similar radiation patterns like an ordinary monopole structure. The observation that both of them are supporting slow-wave propagation, leads to a modification of the propagation constants which in turn is used to model the input characteristics of the original structures. 
For completeness the analysis of a CPW-fed strip monopole radiator is briefly presented in Sec. 2.1 [15]. The printed zigzag-shaped structure is then considered (Sec. 2.2) which is a planar counterpart of a normal-mode helical antenna [16]. Albeit there are available literature on the impedance characteristics of axial mode zigzag antennas [17], studies on normal mode planar zigzag monopoles are unknown to the authors. Later, the planar corrugated monopole radiator is considered. This structure also supports slow-wave propagation and is modeled as a superposition of four normal-mode zigzag antennas. Circuit theoretic analyses are used to find closed-form expressions for the input characteristics of this configuration. Section 3 presents the simulation results along with those obtained from the measurements and compares the same with the proposed theoretical formulations. The achieved miniaturizations for the two structures are also depicted graphically as compared to single rectangular strip monopoles of the same radiating heights in this section. Radiation patterns of both the antennas are provided to support the claim of the authors that they are radiating in the normal-mode.

\section{Theoretical Formulation}

\subsection{Analysis of the Strip Monopole Structure}

Recently a theoretical investigation on the input characteristics of a strip monopole using induced EMF method has been reported [15]. In the work, to find the expression for near zone electric field intensity radiated by the antenna, the ground plane was considered to be extended to infinity and image theory was used to conceive a dipole structure. This is technically justified as the CPW-grounds are adequately large compared to the total length of the central line and the slots separating it from the grounds, and such assumptions are also used in numerical techniques and full-wave analysis as cited in [11]. The configuration along with the dipole and the necessary coordinate system are shown in Fig. 1. The total value of $E_{\mathrm{Z}}$ on the Z-axis becomes [12], [13],

$$
\begin{aligned}
E_{\mathrm{Z}}= & -\frac{\mathrm{j} \beta I_{\mathrm{m}}}{4 \pi \omega \varepsilon_{\mathrm{eff}}}\left[\frac{\mathrm{e}^{-\mathrm{j} \beta \sqrt{(H-z)^{2}+R^{2}}}}{\sqrt{(H-z)^{2}+R^{2}}}+\frac{\mathrm{e}^{-\mathrm{j} \beta \sqrt{(H+z)^{2}+R^{2}}}}{\sqrt{(H+z)^{2}+R^{2}}}\right. \\
& \left.-2 \cos (\beta H) \frac{\mathrm{e}^{-\mathrm{j} \beta \sqrt{z^{2}+R^{2}}}}{\sqrt{z^{2}+R^{2}}}\right]
\end{aligned}
$$

where $I_{\mathrm{m}}=I_{\mathrm{m}_{\mathrm{L}}}+I_{\mathrm{m}_{\mathrm{R}}}$.

The input impedance of the antenna is given by

$$
Z_{11}=-\frac{K}{I_{\mathrm{m}}} \int_{0}^{H} E_{\mathrm{Z}} \sin [\beta(H-Z)] \mathrm{d} Z ; K=\frac{1}{\sin ^{2}(\beta H)} .
$$

Substituting the value of $E_{\mathrm{Z}}$ from (1) in (2) the resistive and reactive parts of the input impedance are expressed in terms of cosine and sine integrals as follows [18], [19].

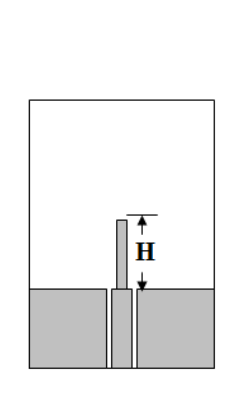

(a)

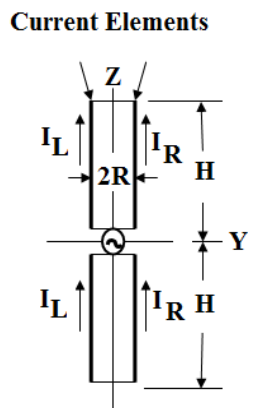

(b)
Fig. 1. (a) Top view of the printed strip monopole, (b) the corresponding dipole with the coordinate system.

$R_{11}=\frac{15 K}{\sqrt{\varepsilon_{\text {eff }}}}$
$\left[\begin{array}{l}\sin (2 \beta H)\left\{\operatorname{Si}\left(u_{2}\right)-\operatorname{Si}\left(v_{2}\right)-2 \operatorname{Si}\left(v_{1}\right)+2 \operatorname{Si}\left(u_{1}\right)\right\} \\ -\cos (2 \beta H) \\ \quad\left\{2 \mathrm{Ci}\left(u_{1}\right)-2 \mathrm{Ci}\left(u_{0}\right)+2 \mathrm{Ci}\left(v_{1}\right)-\operatorname{Ci}\left(u_{2}\right)-\operatorname{Ci}\left(v_{2}\right)\right\} \\ -2\left\{\operatorname{Ci}\left(u_{1}\right)-2 \mathrm{Ci}\left(u_{0}\right)+\operatorname{Ci}\left(v_{1}\right)\right\}\end{array}\right]$

$X_{11}=-\frac{15 K}{\sqrt{\varepsilon_{\text {eff }}}}$

$\left[\begin{array}{l}\operatorname{Sin}(2 \beta H)\left\{2 \operatorname{Ci}\left(v_{1}\right)-2 \operatorname{Ci}\left(u_{1}\right)+\operatorname{Ci}\left(v_{2}\right)-\operatorname{Ci}\left(u_{2}\right)\right\} \\ -\operatorname{Cos}(2 \beta H) \\ \quad\left\{2 \operatorname{Si}\left(u_{1}\right)-2 \operatorname{Si}\left(u_{0}\right)+2 \operatorname{Si}\left(v_{1}\right)-\operatorname{Si}\left(u_{2}\right)-\operatorname{Si}\left(v_{2}\right)\right\} \\ -2\left\{\operatorname{Si}\left(u_{1}\right)-2 \operatorname{Si}\left(u_{0}\right)+\operatorname{Si}\left(v_{1}\right)\right\}\end{array}\right]$

where

$$
\begin{gathered}
u_{0}=\beta R, \quad u_{1}=\beta\left(\sqrt{R^{2}+H^{2}}-H\right), \\
v_{1}=\beta\left(\sqrt{R^{2}+H^{2}}+H\right) ; \\
u_{2}=\beta\left(\sqrt{R^{2}+(2 H)^{2}}+2 H\right), \\
v_{2}=\beta\left(\sqrt{R^{2}+(2 H)^{2}}-2 H\right) .
\end{gathered}
$$

Expressions for the input impedance of the configuration shown in Fig. 1(a) are calculated by modeling the CPW line as an equivalent lossless transmission line having characteristic impedance equal to $R_{0}$. The input impedance of this assembly is expressed as [20]

$$
Z_{\mathrm{IN}}=R_{0}\left[\frac{Z_{\mathrm{A}}+\mathrm{j} R_{0} \tan (\beta L)}{R_{0}+\mathrm{j} Z_{\mathrm{A}} \tan (\beta L)}\right]
$$

where $Z_{\mathrm{A}}$ is the input impedance of the antenna, $R_{0}$ is the characteristic impedance of the $\mathrm{CPW}$ line, and $L$ is the length of the line.

\subsection{Analysis of the Normal Mode Zigzag Radiator}

A zigzag monopole antenna along with a CPW feed is printed on an $A \times B$ dielectric substrate as shown in Fig. 2(a). 


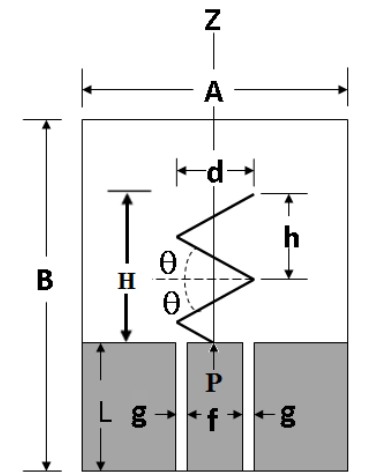

(a)

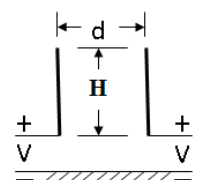

(b)

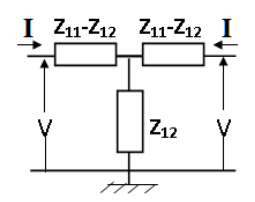

(c)
Fig. 2. (a) A zigzag monopole antenna, (b) the equivalent dipole model and (c) its circuit representation.

The width of the antenna is $d$ and the pitch is $h$. The pitch angle of the structure is denoted by $\theta$. Phase velocity along the zigzag conductor is considered to be equal to the velocity of light in the substrate - calculated using its effective dielectric constant. This assumption is used to obtain the phase velocity along Z-axis [21], [22], and the propagation constant $\beta_{\text {mod }}$ along the same as in (6) and (7) respectively

$$
\begin{gathered}
v_{\mathrm{zp}}=c \sin \theta, \\
\beta_{\mathrm{mod}}=\frac{2 \pi}{\lambda_{\mathrm{g}}}
\end{gathered}
$$

where $\lambda_{\mathrm{g}}=\mathrm{v}_{\mathrm{zp}} / f$ and $\omega=f /(2 \pi)$.

As a first order approximation, the zigzag structure is considered as a rectangular strip monopole supporting a slow wave with the modified propagation constant $\beta_{\text {mod. }}$. With the symbols having their usual meanings, expressions for the resistance and the reactance of the zigzag monopole structure are derived from (3), (4) as (8), (9),

$$
\begin{aligned}
& R_{11}=\frac{15 K}{\sin \theta \sqrt{\varepsilon_{\text {eff }}}} \\
& {\left[\begin{array}{l}
\sin \left(2 \beta_{\text {mod }} H\right)\left\{\operatorname{Si}\left(u_{2}\right)-\operatorname{Si}\left(v_{2}\right)-2 \operatorname{Si}\left(v_{1}\right)+2 \operatorname{Si}\left(u_{1}\right)\right\} \\
-\cos \left(2 \beta_{\text {mod }} H\right) \\
\left\{2 \operatorname{Ci}\left(v_{1}\right)-2 \operatorname{Ci}\left(u_{0}\right)-\operatorname{Ci}\left(u_{2}\right)+2 \operatorname{Ci}\left(u_{1}\right)-\operatorname{Ci}\left(v_{2}\right)\right\} \\
+4 \operatorname{Ci}\left(u_{0}\right)-2 \operatorname{Ci}\left(v_{1}\right)-2 \operatorname{Ci}\left(u_{1}\right)
\end{array}\right],}
\end{aligned}
$$

$$
X_{11}=-\frac{15 K}{\sin \theta \sqrt{\varepsilon_{\text {eff }}}}
$$$$
\left[\begin{array}{l}
\sin \left(2 \beta_{\text {mod }} H\right)\left\{2 \operatorname{Ci}\left(v_{1}\right)-\operatorname{Ci}\left(u_{2}\right)-2 \operatorname{Ci}\left(u_{1}\right)+\operatorname{Ci}\left(v_{2}\right)\right\} \\
-\operatorname{Cos}\left(2 \beta_{\text {mod }} H\right) \\
\quad\left\{2 \operatorname{Si}\left(v_{1}\right)-2 \operatorname{Si}\left(u_{0}\right)-\operatorname{Si}\left(u_{2}\right)-\operatorname{Si}\left(v_{2}\right)+2 \operatorname{Si}\left(u_{1}\right)\right\} \\
+4 \operatorname{Si}\left(u_{0}\right)-2 \operatorname{Si}\left(u_{1}\right)-2 \operatorname{Si}\left(v_{1}\right)
\end{array}\right]
$$

where $u_{0}=\beta_{\text {mod }}\left(\frac{d}{2}\right), u_{1}=\beta_{\text {mod }}\left[\sqrt{\left(\frac{d}{2}\right)^{2}+H^{2}}-H\right]$,

$$
\begin{aligned}
& v_{1}=\beta_{\text {mod }}\left[\sqrt{\left(\frac{d}{2}\right)^{2}+H^{2}}+H\right] ; \\
& u_{2}=\beta_{\text {mod }}\left[\sqrt{\left(\frac{d}{2}\right)^{2}+(2 H)^{2}}+2 H\right], \\
& v_{2}=\beta_{\text {mod }}\left[\sqrt{\left(\frac{d}{2}\right)^{2}+(2 H)^{2}}-2 H\right] .
\end{aligned}
$$

Direct use of the expressions, however, is not desired because the width of the equivalent strip $d$ is fairly large. A close look reveals that the Z-components of the current element that makes an acute angle with the horizontal line is located along the left side of the modeled rectangular strip. Other set of inclined current elements making an obtuse angle with the horizontal line will contribute to a set of $\mathrm{Z}$ directed current elements concentrated at the right side of the conceived rectangular strip. Each set of vertical current elements is approximated to a single line current which in turn is considered as an imaginary monopole. This assumption permits a configuration consisting of a pair of parallel monopoles of height $H$ ( $H$ being the physical height of the zigzag structure) separated by a distance $d$ and fed by the same source, to model the original structure. The model is shown in Fig. 2(b) and its circuit theoretic representation is shown in Fig. 2(c), where $Z_{11}$ is the self-impedance of the constituent monopoles and $Z_{12}$ is the mutual impedance between them. Expressions (8) and (9) can be simultaneously used by replacing $d / 2$ with $d$, which is the gap between the equivalent constituent monopoles, to determine the values of the mutual impedances [23]. The width of the individual imaginary monopoles is considered to be very small and a value of $d=.005 \mathrm{~mm}$ is used to calculate the corresponding self impedances.

Since both the constituent monopoles are being excited using the same voltage source, the series impedances in Fig. 2(c) can be connected in parallel as shown in Fig. 3 [24]. From Fig. 3 one can easily obtain the expression for the input impedance of the zigzag structure at the point $\mathrm{P}$ of Fig. 2(a),

$$
Z_{\mathrm{A}}=\frac{Z_{11}+Z_{12}}{2}
$$

Correspondingly, the input impedance at the CPW-feeding port of the structure is obtained by translating the impedance along the length $L$ of a $50 \Omega$ transmission line using (5).

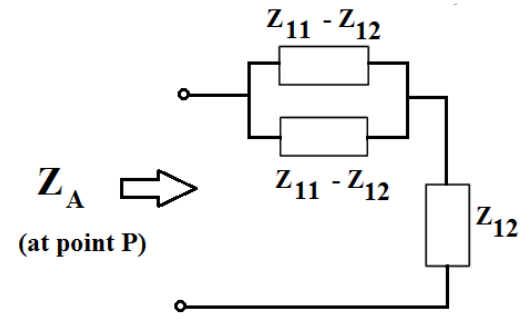

Fig. 3. Simplified circuit model to evaluate the input impedance. 


\subsection{Analysis of the Corrugated Monopole Antenna}

This analysis is motivated by the concept depicted in [20], [25], [26] where a single turn of a helix was considered as a combination of a vertical conductor and a loop connected in series. In this paper a reverse concept is used, that is, a vertical and horizontal current element forming an inverted $\mathrm{L}$ are combined to get an inclined current carrying conductor as shown in Fig. 4(b). The corrugated planer monopole antenna is shown in Fig. 4(a). For simplicity it is considered that the corrugated strips of the configuration are made of very thin lines, that is, $w \approx 0$. The simplified corrugated part is shown in Fig. 4(c).

It is to be noted that, according to the simulations, the antenna was designed to resonate around $5 \mathrm{GHz}$. Before proceeding any further, the current directions on the original structure are investigated using the simulations on ANSYS HFSS [27]. Figure 5 shows the simulated current directions on the proposed corrugated structure.

For the purpose of theoretical analysis it is assumed that $w \approx 0$. From the current distribution shown in Fig. 5 it is observed that the horizontal arms support current elements in two different directions, viz., towards and away from the vertical arm. These two sets of current elements suggest two different corrugated structures as shown in Fig. 6(a). Each such component may be bifurcated as shown in Fig. 6(b).

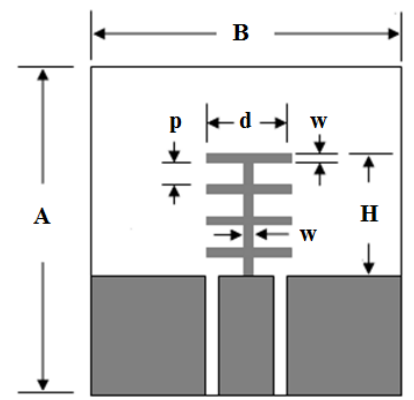

(b)

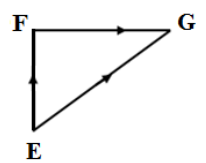

(c)

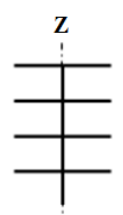

(a)

Fig. 4. (a) Planer corrugated monopole antenna configuration; (b) an inverted L shaped current element and its equivalent inclined counterpart; and (c) the thin corrugated structure.

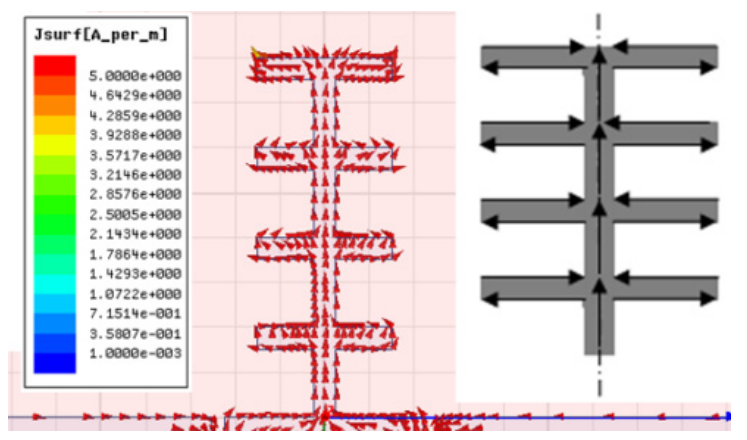

Fig. 5. Simulated current distribution on the proposed structure at $5.15 \mathrm{GHz}$ and the highlighted currents in the proposed schematic.

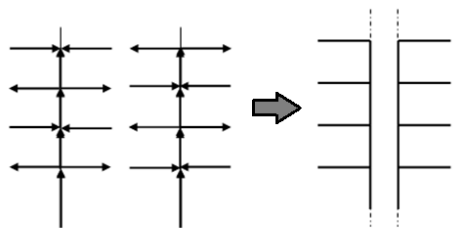

(a)

(b)

Fig. 6. Two components of the original structure and their possible bifurcation.

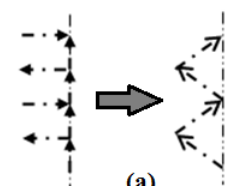

(a)

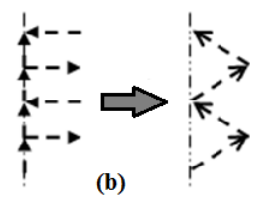

(b)

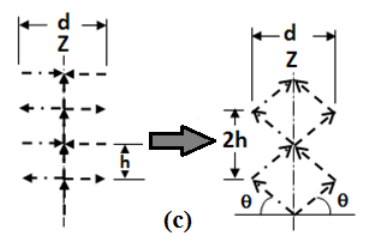

Fig. 7. Equivalent zigzag representations of the first corrugated component.

Considering first the left corrugated component of Fig. 6(a) and using the equivalence described in Fig. 4(a), each bifurcated part may be represented by a zigzag formation as shown in Fig. 7(a) and (b). Figure 7(c) shows the equivalent model of the corrugated antenna using two zigzag patterns.

Similar arguments will derive the equivalent representation of the other corrugated component as shown in Fig. 8. From the above discussion it may be noted that if the original antenna is bisected into its left and right counterparts then each part can be represented by a pair of equivalent zigzag structure and by the argument presented in Sec. 2.2 each such zigzag structure can be represented by a strip monopole antenna. The axis of this pair being identical they may be represented by a single strip monopole structure. This leads to the equivalent representation of the original corrugated monopole into a pair of strip monopole antenna fed from a single source.

Assuming the pitch angle of the constituent zigzag structures to be $\theta$; and the phase velocity along the zigzag conductor equal to the velocity of light in the substrate - calculated using its effective dielectric constant - one may utilize equations (6), (7) to calculate the phase velocity along the $\mathrm{Z}$-axis as $v_{\mathrm{zp}}$ and the modified propagation

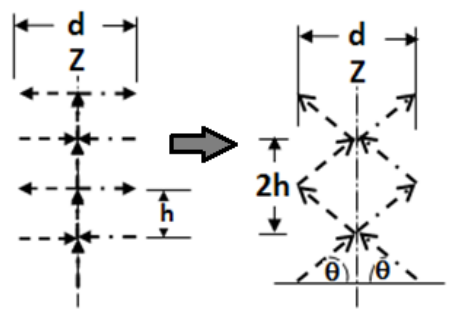

Fig. 8. Equivalent zigzag representation of the second corrugated component. 


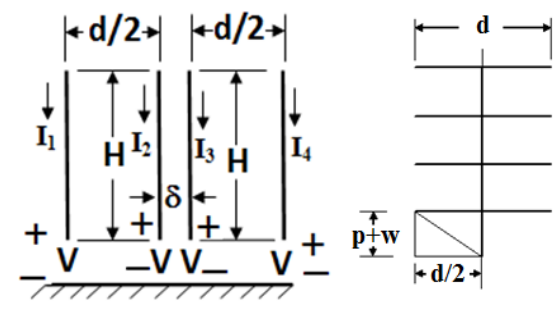

(a)

(b)

Fig. 9. (a) Equivalent strip monopole representation for the corrugated structure. (b) Equivalent thin corrugated structure.

constant $\beta_{\text {mod, }}$ required for the formulations. Equivalent representation for the present structure, therefore, should contain four such thin strip monopoles of height $H=4 h$ as shown in Fig. 9(a).

The current voltage relations in the conceived antennas in Fig. 9(a) is represented by the same technique as described during the analyses in Sec. 2.2 as

$$
\left[\begin{array}{l}
V \\
V \\
V \\
V
\end{array}\right]=\left[\begin{array}{llll}
Z_{11} & Z_{12} & Z_{13} & Z_{14} \\
Z_{21} & Z_{22} & Z_{23} & Z_{24} \\
Z_{31} & Z_{32} & Z_{33} & Z_{34} \\
Z_{41} & Z_{42} & Z_{43} & Z_{44}
\end{array}\right]\left[\begin{array}{c}
I_{1} \\
I_{2} \\
I_{3} \\
I_{4}
\end{array}\right] .
$$

Since two zigzag antennas represent the bifurcated portions of the corrugated monopole, the gap $\delta$ between the second and the third antenna should be very small and in the limit it should tend to zero. With this assumption one gets,

$Z_{11}=Z_{22}=Z_{33}=Z_{44}=Z_{23}=Z_{32}=Z_{1}$,

$Z_{12}=Z_{21}=Z_{13}=Z_{31}=Z_{24}=Z_{42}=Z_{34}=Z_{43}=Z_{2}$,

$Z_{14}=Z_{41}=Z_{3}$,

i.e. $Z_{1}$ is the self-impedance of a thin strip monopole of height $H, Z_{2}$ is the mutual impedance between two thin strip monopoles of height $H$ and separation $d / 2, Z_{3}$ is mutual impedance between two thin strip monopoles of height $H$ and separation $d$. Solving (11) one gets,

$$
Z_{\mathrm{A}}=\frac{V}{I_{1}+I_{2}+I_{3}+I_{4}}=\frac{4 Z_{2}^{2}-2 Z_{1}^{2}-2 Z_{1} Z_{3}}{8 Z_{2}-6 Z_{1}-Z_{3}} .
$$

From the parameters explained in Fig. 4 the equivalent thin corrugated structure is shown in Fig. 9(b) and the modified propagation constant for the structure is given by

$$
\beta_{\text {mod }}=\frac{2 \pi}{\lambda_{\mathrm{g}}} \frac{\sqrt{\left(\frac{d}{2}\right)^{2}+(p+w)^{2}}}{p+w} .
$$

Equations (11), (13), (14) along with (5), (8-10) can be utilized to find the input characteristics of the corrugated monopole radiator. The following section will discuss and compare the simulated and measured results with the theoretical predictions.

\section{Results and Analyses}

In the last section theoretical analysis for three different monopole structures, viz., strip monopole shown in Fig. 1, zigzag monopole shown in Fig. 2 and corrugated monopole shown in Fig. 4 has been made. In this section these formulations will be used to estimate the possible miniaturizations achievable from these structures. Arlon AD430 with dielectric constant 4.3 and thickness of $0.762 \mathrm{~mm}$ is used as substrate for all subsequent applications. In Fig. 10(a) input impedance of a zigzag antenna is compared with that of a strip dipole antenna with the same height and width. Similar comparison for corrugated monopole is made in Fig. 10(b). It is to be noted that for the zigzag-shaped normal-mode structure the degree of miniaturization is more compared to that for the corrugated configuration.

A prototype of the zigzag-shaped normal-mode radiator (as shown in Fig. 2) is fabricated with the dimensions $A=B=35 \mathrm{~mm}, \quad h=4 \mathrm{~mm}, \quad d=3 \mathrm{~mm}, \quad g=0.3 \mathrm{~mm}$, $f=4.46 \mathrm{~mm}, L=10 \mathrm{~mm}, H=7 \mathrm{~mm}$ as in Fig. 11 and the width of the zigzag strip is taken as $0.3 \mathrm{~mm}$ respectively. The feed-width of the CPW-excitation, to maintain $50 \Omega$ characteristic impedance, is calculated using the IE3D Line Gauge Calculator [28].

Figure 12(a) includes the plots of experimental and the theoretical input impedances of the fabricated structure. The figure shows that the resonating frequencies for both the theoretical and the experimental plots are almost the same. Figure 12(b) describes the comparison between the theoretical and the measured results of $\mathrm{S}_{11}$. Considering the

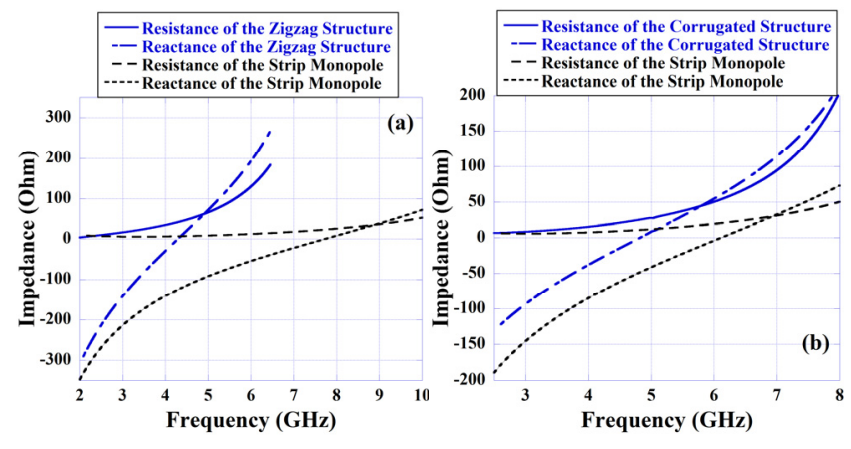

Fig. 10. (a) Impedance plots of the zigzag shaped structure and a corresponding strip monopole radiator (both of total height $H=7 \mathrm{~mm}$ and width $=3 \mathrm{~mm}$ ). (b) Impedance plots of the corrugated monopole structure and a corresponding strip monopole radiator (both of total height $H=8 \mathrm{~mm}$ and width $=3 \mathrm{~mm}$ ).

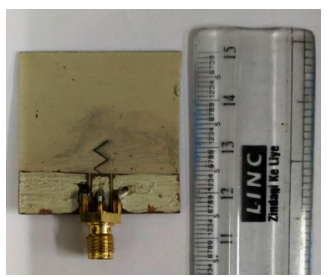

Fig. 11. The fabricated prototype of the normal-mode zigzag structure. 

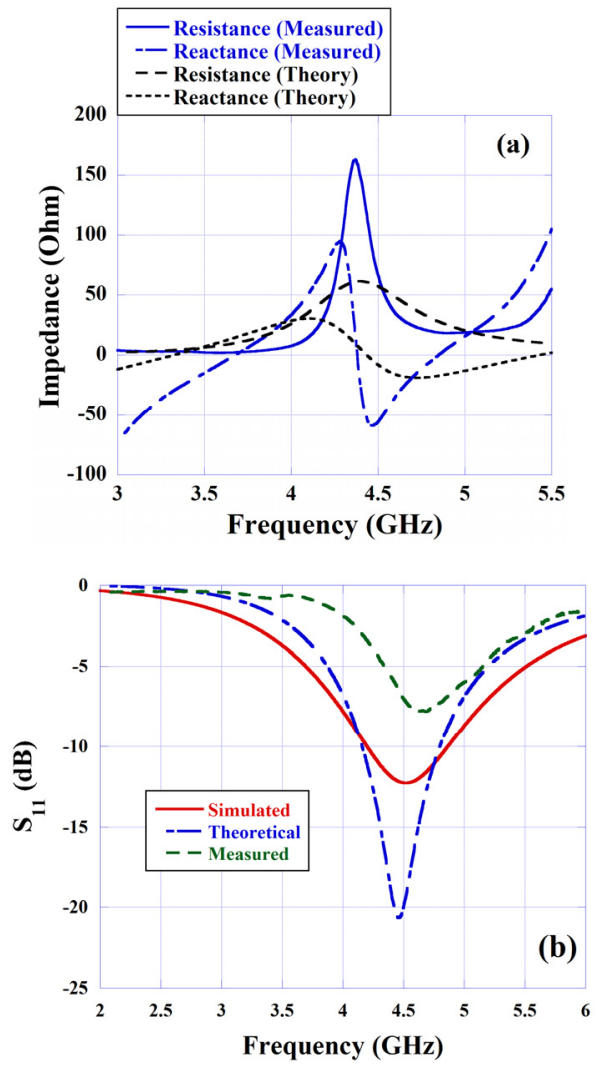

Fig. 12. (a) The comparison of the theoretical and the experimental values of the input impedance. (b) The comparison of the theoretical, simulated and the experimental values of $\mathrm{S}_{11}$ for the zigzag-shaped normalmode radiator.
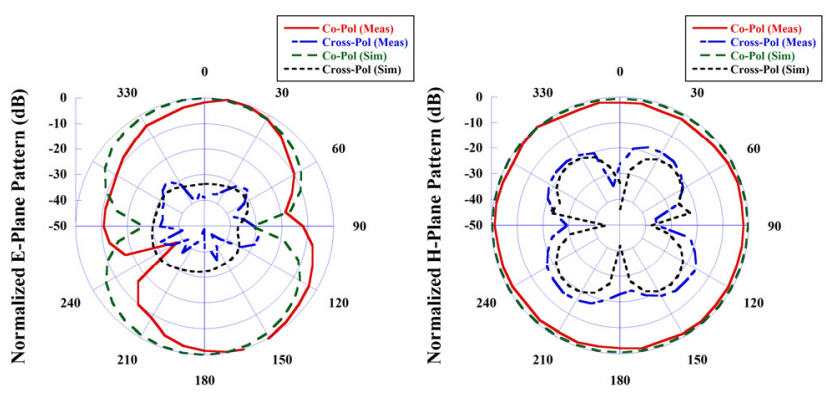

Fig. 13. Measured (a) E-plane and (b) H-plane radiation patterns for the normal-mode zigzag-shaped radiator at $4.53 \mathrm{GHz}$.

size of the structure, which is electrically comparable to the size of the available connectors, one has to cater for the probe inaccuracies by taking into account their reactances [29]. Therefore a small inductance of $0.48 \mathrm{nH}$ is assumed to be connected in series to the antenna configuration and the theoretical results are compared with the final measurements accordingly.

The measured frequency of resonance for the zigzagshaped structure is $4.53 \mathrm{GHz}$ whereas; the predicted frequency of resonance is obtained at $4.42 \mathrm{GHz}$ with that of the simulations at $4.51 \mathrm{GHz}$. Further theoretical experimentation suggests that the discrepancies in amplitudes may have existed due to some error in fabrication that may generate a higher characteristic impedance of the CPW feed. Moreover the difference might also be possible due to the heuristics used in the theoretical modeling procedure. The claim of the work lies in the fact that not only it has succeeded to predict the resonant frequency with a high accuracy, but also the nature of the predicted curves shows a good match with the measured ones. It can also be mentioned here that the measured radiation patterns of the structure, as shown in Fig. 13(a, b), support the claim that it radiates in the normal mode. The measured maximum realized gain of the zigzag structure was found to be $0.672 \mathrm{~dB}$ at $4.53 \mathrm{GHz}$.

To analyze the corrugated monopole structure as in Fig. 4, a design to resonate around $5 \mathrm{GHz}$ band is simulated and optimized using ANSYS HFSS. The optimization was carried out to fix the width $d$ and the pitch $p$ of the corrugated strips as in Fig. 4. Figures 14(a, b) give the optimization results and compare them with their corresponding theoretical predictions - which show a near-perfect match of the resonance frequencies as well as the nature of the plots. A prototype of the corrugated monopole antenna is fabricated on the same substrate material having a relative dielectric constant of 4.3 and a thickness of $0.762 \mathrm{~mm}$ having the dimensions as $A=20 \mathrm{~mm}$, $B=20 \mathrm{~mm}, p=1.5 \mathrm{~mm}, d=3 \mathrm{~mm}$ and $w=0.5 \mathrm{~mm}$ respectively.

The fabricated structure is shown in Fig. 15. Figure 16 depicts the comparison of the simulated, measured and theoretically predicted results for $\mathrm{S}_{11}$ of the structure. The results show an excellent match for the frequency of resonance, thus establishing the strength of the proposed theory in this work - to accurately predict the resonant frequencies of slow-wave supporting planar monopole antennas on dielectrics. Impedance bandwidth of the structure as measured - it also matches well with the theoretical predictions as seen. Figures $17(\mathrm{a}, \mathrm{b})$ give the measured radiation patterns of the corrugated monopole antenna for the $\mathrm{E}$ and the $\mathrm{H}$ planes respectively. Measured radiation patterns for both the antennas show expected behavior with minor anomalies, probably due to the limitations of the measurement setup. The maximum realized gain of the corrugated structure is measured to be $0.973 \mathrm{~dB}$ at $5.15 \mathrm{GHz}$.
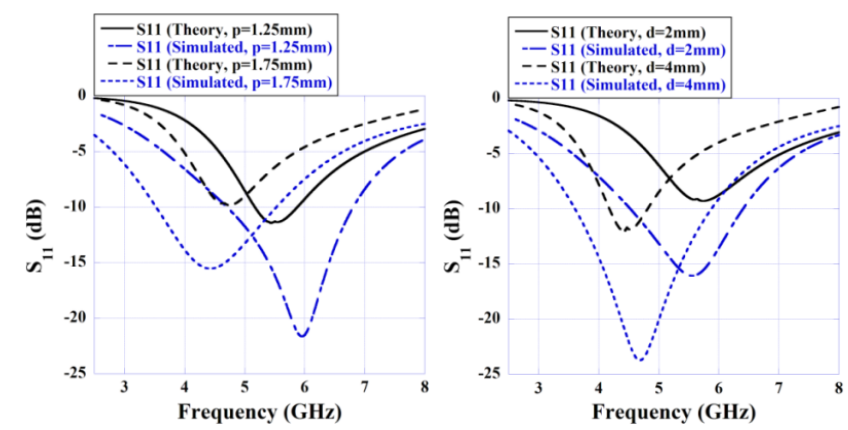

Fig. 14. Optimized results for $S_{11}$ and their comparisons with their corresponding theoretical predictions for (a) pitch $p$ and (b) width $d$ of the corrugated monopole structure. 


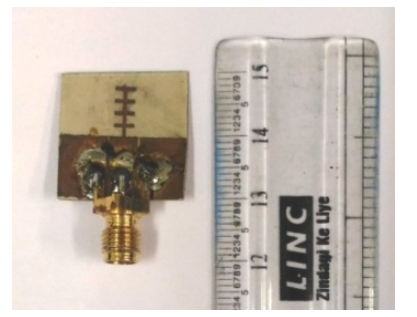

Fig. 15. The fabricated prototype of the corrugated monopole antenna.

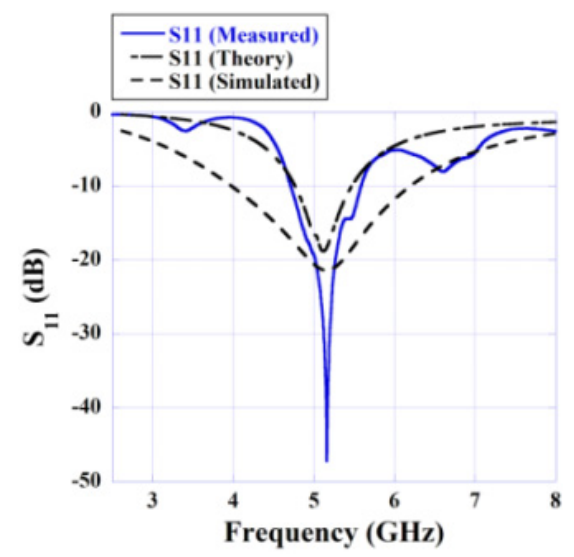

Fig. 16. The comparison of the proposed resonant frequency with the simulation and the measured results.
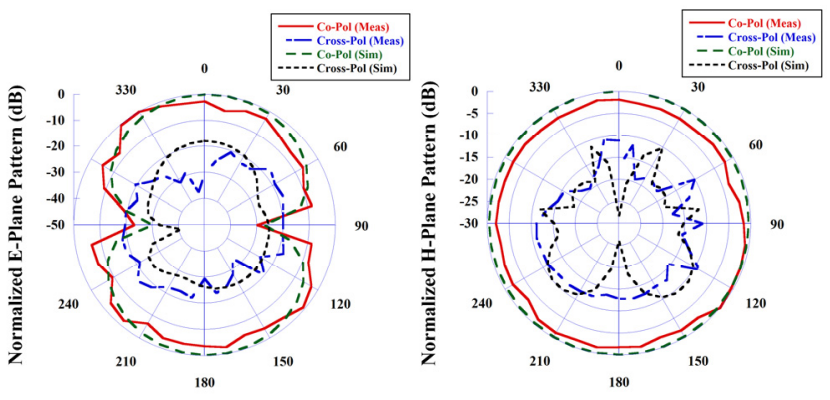

Fig. 17. Measured (a) E-plane and (b) H-plane radiation patterns for the corrugated monopole radiator at $5.15 \mathrm{GHz}$.

Both the antennas are found to be low-gain radiators. This is so because both of them support slow-wave propagation and thereby resonate at small physical dimensions with near-omnidirectional radiation patterns. It may be noted that even for a $\mathrm{CPW}$-fed rectangular strip monopole, it exhibits similar radiation characteristics [30].

\section{Conclusions}

The paper presents some novel theoretical approaches to establish closed-form expressions of the input-impedance of CPW-Fed planar strip monopole antennas. Effective miniaturization techniques, such as meandering and using corrugated strips on the monopole to support slow waves, are used to design a zigzag-shaped normal-mode radiator and a corrugated monopole antenna. Appropriate theories are proposed to predict the corresponding resonant frequencies. The simulations, as well as the measured datasets are compared with the theoretical predictions so as to validate the effectiveness of the circuit model under practical circumstances. It may be noted that the theoretical value of $S_{11}$ gives better result in case of the corrugated structure than the zigzag monopole. In this paper a zigzag antenna is represented by a rectangular strip monopole supporting slow wave propagation. The accuracy of such model is better for zigzag structure with small width. The corrugated antenna could be modeled by a pair of zigzag configuration with half of the width of the original structure. In case of the single zigzag monopole, however, such reduction could not be obtained. This may be the reason for the above discrepancy observed in the resonant frequencies obtained from the theory and the measurements for the concerned radiator. Advantage of this model over full wave numerical techniques and conformal mapping procedures lies in its simplicity and reduced computational requirements. The work proposed here presents closed-form expressions utilizing a circuit approach requiring minimum computing resources as against the available commercial EM solvers, and they do suggest accurate predictions verified by the simulations and practical experiments.

\section{Acknowledgments}

This work is supported by CSIR, Govt. of India (ack. No.: 143126/2K17/1 and File No. 09/096(0925)/2018EMR-I, dated: $02 / 05 / 2018$ ). The authors would also like to thank the anonymous reviewers for their valuable comments and advices on this article.

\section{References}

[1] KIM, J., RAHMAT-SAMII, Y. Planar inverted-F antennas on implantable medical devices: Meandered type versus spiral type. Microwave and Optical Technology Letters, 2006, vol. 48, no. 3, p. 567-572. DOI: $10.1002 / \mathrm{mop} .21409$

[2] KARACOLAK, T., HOOD, A. Z., TOPSAKAL, E. Design of a dual-band implantable antenna and development of skin mimicking gels for continuous glucose monitoring. IEEE Transactions on Microwave Theory and Techniques, 2008, vol. 56, no. 4, p. 1001-1008. DOI: 10.1109/TMTT.2008.919373

[3] LIU, W. C., GHAVAMI, M., CHUNG, W. C. Triple-frequency meandered monopole antenna with shorted parasitic strips for wireless application. IET Microwaves, Antennas and Propagation, 2009, vol. 3, no. 7, p. 1110-1117. DOI: 10.1049/ietmap.2008.0204

[4] LIU, W. C., CHEN, Y. C. Compact strip-monopole antenna for WLAN-band USB dongle application. Electronics Letters, 2011, vol. 47, no. 8, p. 479-480. DOI: 10.1049/el.2010.3234

[5] ZUO, S. L., ZHANG, Z., Y., YANG, J. W. Planar meander monopole antenna with parasitic strips and sleeve feed for DVB$\mathrm{H} / \mathrm{LTE} / \mathrm{GSM} 850 / 900$ operation in the mobile phone. IEEE Antennas and Wireless Propagation Letters, 2013, vol. 12, p. 27-30. DOI: 10.1109/LAWP.2012.2234717 
[6] MAURYA, N. K., BHATTACHARYA, R. CPW-fed dual-band pseudo-monopole antenna for LTE/WLAN/WiMAX with its usage in MIMO. In IEEE International Symposium on Antennas and Propagation (APSURSI). Fajardo (Puerto Rico), 2016. DOI: 10.1109/APS.2016.7695936

[7] BHATTACHARYA, R., GARG, R., BHATTACHARYA, T. K. A compact Yagi-Uda type pattern diversity antenna driven by CPW-fed pseudomonopole. IEEE Transactions on Antennas and Propagation, 2016, vol. 64, no. 1, p. 25-32. DOI: 10.1109/TAP.2015.2499756

[8] WANG, C. J., HSIAO, K. L. CPW-fed monopole antenna for multiple system integration. IEEE Transactions on Antennas and Propagation, 2014, vol. 62, no. 2, p. 1007-1011. DOI: 10.1109/TAP.2013.2290551

[9] ESTARKI, M. D., VAUGHAN, R. G. Theoretical methods for the impedance and bandwidth of the thin dipole. IEEE Antennas and Propagation Magazine, 2013, vol. 55, no. 1, p. 62-81. DOI: 10.1109/MAP.2013.6474485

[10] KOLUNDZIJA, B. M., OGNJANOVIC, J. S., SARKAR, T. K WIPLD: Electromagnetic Modeling of Composite Metallic and Dielectric Structures. Norwood (MA): Artech House, 2000. ISBN: 978-0890063583

[11] WOLFF, I. Coplanar Microwave Integrated Circuits. Chapter 2. Wiley Interscience, 2006. ISBN: 978-0-471-12101-5

[12] TAFLOVE, A., UMASHANKAR, K. R. The finite-difference time-domain method for numerical modeling of electromagnetic wave interactions. Electromagnetics, 1990, vol. 10, no. 1, p. 105-126. DOI: $10.1080 / 02726349008908231$

[13] JONES, D. S. Methods in Electromagnetic Wave Propagation. Oxford: Clarendon Press, 1979.

[14] LEE, Y. H., JUNG, J. H., PARK, I. Multiple meander strip monopole antenna. Electronics Letters, 2005, vol. 41, no. 7, p. 391-393. DOI: 10.1049/el:20058255

[15] BANERJEE, A., BANDYOPADHYAY, A. K. Theoretical investigation on the input impedance of a CPW-fed strip monopole antenna. Microwave and Optical Technology Letters, 2017, vol. 59, no. 2, p. 346-348. DOI: 10.1002/mop.30287

[16] HIROI, Y., FUJIMOTO, K. Practical usefulness of normal mode helical antenna. In IEEE Antennas and Propagation Society International Symposium. Amherst (MA, USA), 1976, p. 238-241. DOI: 10.1109/APS.1976.1147656

[17] NAKANO, H., TAGAMI, H., YAMAUCHI, J. Numerical analysis of an axial mode zigzag antenna. Electronics Letters, 1985, vol. 21, no. 14, p. 606-608. DOI: 10.1049/el:19850428

[18] JORDAN, E. C., BALMAIN, K. G. Electromagnetic Waves and Radiating Systems. Prentice-Hall of India (Pvt.), Ltd, 1964. ISBN: 0132499959

[19] ROY, M. N., BANDYOPADHYAY, A. K. Input impedance and radiation efficiency of a center-fed dipole antenna with feed points displaced transverse to dipole axis. Proc. IEEE (Letters), 1969, vol. 57 , no. 7 , p. 1344-1345. DOI: 10.1109/PROC.1969.7270

[20] KRAUS, J. D. Antennas. McGraw-Hill, 1950. ISBN: 0070354227

[21] RYDER, J. D. Networks, Lines and Fields. New Delhi: Prentice Hall, 2003. ISBN: 978-8120302990

[22] WALDRON, R. A. A helical coordinate system and its applications in electromagnetic theory. The Quarterly Journal of Mechanics and Applied Mathematics, 1958, vol. 11, no. 4, p. 438-461. DOI: $10.1093 /$ qjmam/11.4.438

[23] KOMPFNER, R. Travelling-wave tubes. Reports on Progress in Physics, 1952, vol. 15, p. 275-327. DOI: 10.1088/0034$4885 / 15 / 1 / 309$
[24] BANERJEE, A, CHATTERJEE, S., GUPTA, B., BANDYOPADHYAY, A. K. Theoretical investigation on input characteristics of CPW-fed wide rectangular monopole structures. In 2017 IEEE International Conference on Antenna Innovations and Modern Technologies for Ground, Aircraft and Satellite Applications (iAIM). Bangalore (India), November 2017. DOI: 10.1109/IAIM.2017.8402614

[25] WHEELER, H. A. A helical antenna for circular polarization. Proceedings of the IRE, 1947, vol. 35, no. 12, p. 1484-1488. DOI: 10.1109/JRPROC.1947.234573

[26] KRAUS, J. D. The helical antenna. Proceedings of the IRE, 1949 , vol. 37, no. 3, p. 263-272. DOI: 10.1109/JRPROC.1949.231279

[27] Ansoft Corp HFSS v.13

[28] Zealand Corp IE3D v.10

[29] HARRINGTON, R. F. Time-Harmonic Electromagnetic Fields. New York: McGraw-Hill, 1961. ISBN: 0-471-20806-X

[30] LAOHAPENSAENG, C., FREE, C., ROBERTSON, I. D. Simplified analysis of printed strip monopole antenna fed by a CPW. In Proceedings of the Asia-Pacific Microwave Conference. Suzhou (China), 2005. DOI: 10.1109/APMC.2005.1606956

\section{About the Authors ...}

Amartya BANERJEE (corresponding author) was born in September, 1992. He received his M.Tech. from IIEST, Shibpur in 2016. His research interests include microwave and antenna design techniques, circuit theoretical analysis and signal processing.

Kaushik PATRA was born in March, 1988. He received his M.E. from Jadavpur University, Kolkata in 2012. His research interests include approximate modeling for microstrip lines for EMC calculation and application in travelling wave and leaky wave antennas.

Sayan CHATTERJEE was born in Kolkata, India, in 1980. Presently, he is an Associate Professor at the Department of Electronics and Telecommunication Engineering, Jadavpur University. His research interest includes microwave and millimeter wave antennas, passive devices and slotted array antenna

Bhaskar GUPTA was born in Kolkata, India, in 1960. He received the B.E., M.E., and Ph.D. degrees from Jadavpur University, Kolkata, India, in 1982, 1984, and 1996, respectively. He is currently a Professor in the Dept. of ETCE, Jadavpur University. His present area of interest is planar antennas, dielectric resonator antennas, wearable antennas, computational electromagnetic, MEMS design and application of soft computing techniques in microwave engineering. He has been named in the 2009 edition of Marquis' Who's Who of the World.

Anup Kumar BANDYOPADHYAY is a retired professor from the Dept. of ETCE, Jadavpur University, Kolkata. His research interests include microwave and antenna engineering, circuit theory and program proving. 